\title{
'This is my vocation; is it worth it?' Why do core medical trainees break from training?
}

\author{
Authors: Matthew Roycroft, ${ }^{A}$ Juan Abad-Madroñero, ${ }^{B}$ Charlotte Cochrane, ${ }^{B}$ Alan Poots, ${ }^{B}$ Mariann Novak, ${ }^{B}$ \\ Amy Tallett, ${ }^{B}$ Sarah-Ann Burger ${ }^{B}$ and Michael FitzPatrick ${ }^{C}$
}

\section{Introduction}

The number of trainees moving directly into training posts following their foundation training is decreasing ${ }^{1}$ and the same pattern is emerging after CMT. Previous studies identified factors behind CMTs leaving the formal training route, but didn't explore the motivations behind this. ${ }^{2,3}$

Understanding the reasons why trainees take a break from traditional training trajectories is crucial in order to ensure structures are embedded to support them. This study investigates trainees' motivations in taking a break after CMT and develops recommendations to support trainees.

\section{Methods}

60-minute semi-structured, recorded, anonymised and transcribed telephone interviews with nine CMTs (five women and four men working in four different deaneries) who self-identified as leaving or taking a break from training were carried out between February and July 2019.

Transcripts were analysed and an emergent thematic framework created. For areas of disagreement we created thematic maps and calculated Cohen's Kappa (0.73), reaching a solid agreement level. ${ }^{4}$ We reached saturation, ${ }^{5}$ when no additional themes were added to the thematic framework, after the second coding of the first six interviews.

\section{Results and analysis}

Three inter-related overarching themes (Fig 1) were identified. Key quotes are shown for each theme.

\section{Personal life}

‘...Last year I felt very burnt out. I was considering leaving medicine completely. So I was looking for other jobs outside medicine and I thought it would be best if I could get a role outside the training programme with reduced hours, to give myself a chance to think if I wanted to stay in a career in medicine...' (Izabelle)

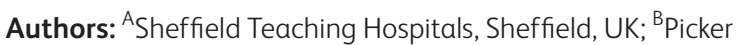
Institute Europe, Oxford, UK; ' CUniversity of Oxford, Oxford, UK

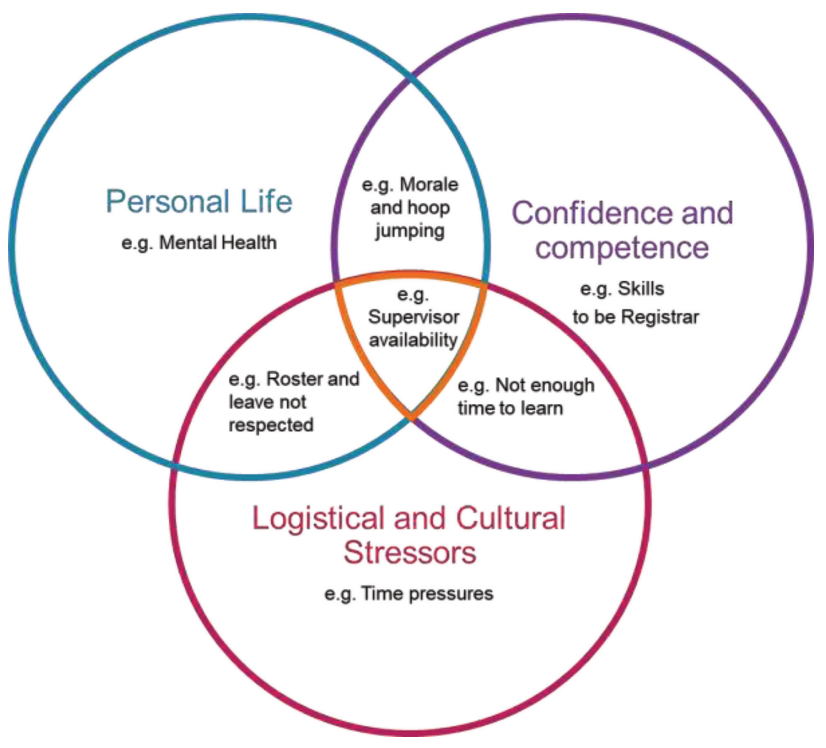

Fig 1. The themes and their inter-relationships for why trainees leave training.

\section{Confidence and competencies}

'I think there needs to be an understanding that [becoming a registrar] doesn't just happen overnight, that I need to be trained to become that role and I need certain competencies. If I don't get those ... and if I don't feel confident enough, then I just won't.' (Finlay)

'I feel like there are some consultants who are so unsupportive' there should be a way of making sure they're not people's supervisors ... I've got Dr so-and-so and everyone grimaces and goes, “Oh no, poor you”, and it shouldn't be like that.' (Jayce)

\section{Logistical and cultural stressors}

'... We're expected to attend 40 clinics over 2 years ... and aren't able to leave work because there's not enough staff ... I came in on annual leave to do [clinics] as well.' (Izabelle)

'Even if you wanted to, and you could step up as a registrar, there would be no one else to do the SHO work.' (Emilis) 


\section{Suggestions to improve training}
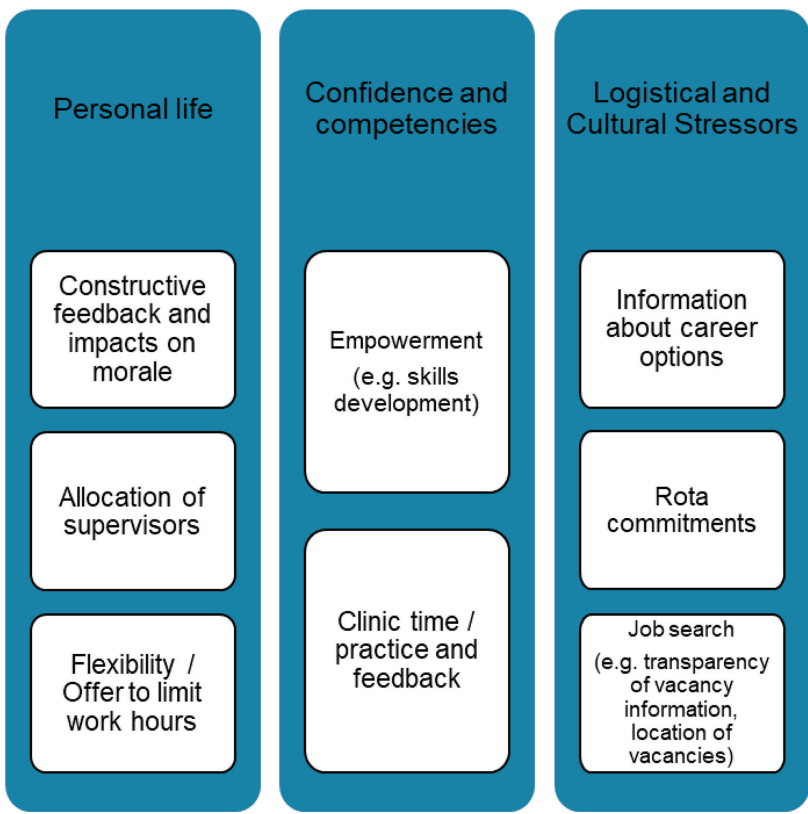

Fig 2. Trainee suggestions for the training programme.

\section{Conclusion}

In addition to the suggestions from interviewees to improve training (Fig 2), this study highlights the need for motivated, well-trained trainers (with the time required for the role) to improve trainee experience. Physicianly specialties need to make flexible training and working more accepted and improve trust understanding of training requirements so they can better support trainees. Flexible Portfolio Training ${ }^{6}$ may help to some degree.
IMT has replaced CMT7 and may address confidence and competence issues by extending the programme and mandating intensive care rotations.

\section{Conflicts of interest}

None declared.

\section{References}

1 Moberly T, Stahl-Timmins W. More doctors are taking a break from training after foundation programme. BMJ 2019;364:1842.

2 Roycroft M, Kaufmann S, Hammond P. Career intention at the end of core medical training. FHJ 2019 Jun;6(Suppl 2):27.

3 Tasker F, Newbery N, Burr B, Goddard AF. Survey of core medical trainees in the United Kingdom 2013 - inconsistencies in training experience and competing with service demands. Clin Med 2014;14:149-56.

4 Burla L, Knierim B, Barth ] et al. From text to codings: intercoder reliability assessment in qualitative content analysis. Nurs Res 2008:57:113-7.

5 Ando H, Cousins R, Young C. Achieving Saturation in Thematic Analysis: Development and Refinement of a Codebook. Comprehensive Psychology 2014:3:1-7.

6 Royal College of Physicians. Flexible portfolio training. London: RCP, 2019 www.rcplondon.ac.uk/projects/flexible-portfolio-training [Accessed 08 October 2019].

7 Joint Royal Colleges of Physicians Training Board. Shape of Training and the physician training model. JRCPTB. www.jrcptb.org.uk/imt [Accessed 09 October 2019]. 